\title{
THE BAADE-WESSELINK METHOD APPLIED TO RR LYRAE VARIABLES IN THE GLOBULAR CLUSTERS M5 AND M92
}

\author{
J. G. COHEN \\ Palomar Observatory, Mail Code 105-24, California Institute of Technology, Pasadena, CA 91125 \\ Received 1992 March 19; accepted 1992 June 4
}

\begin{abstract}
An infrared Baade-Wesselink analysis is given for two RR Lyrae variables in the extremely metal-poor globular cluster M92 and four in the intermediate metallicity cluster M5. The method of analysis relies on the theoretical model atmospheres of Kurucz and on the absolute flux calibration of observed $V$ - and $K$ magnitudes. The best results are obtained with $V-K$ colors to obtain $T_{\text {eff }}$ and $K$-magnitudes to deduce absolute fluxes. We obtain a distance to M5 of $6.9 \pm 0.45 \mathrm{kpc}$ and to M92 of $7.85 \pm 0.45 \mathrm{kpc}$, with a $0.3 \mathrm{kpc}$ additional systematic uncertainty. These results are in excellent agreement with the previously published distances, based on matching the globular cluster main sequence to that defined by the field subdwarfs of known distance. With these distances we obtain an absolute mean magnitude corrected for reddening, $\boldsymbol{M}_{V}^{0}$, of $0.86 \pm 0.14$ mag for the M5 RR Lyrae variables, while $M_{V}^{0}=0.62 \pm 0.11$ for the M92 RR Lyrae variables.

The globular cluster RR Lyrae stars appear to behave just like the field RR Lyrae stars. They obey the same $\left\langle M_{V}\right\rangle-[\mathrm{Fe} / \mathrm{H}]$ relationship as do the field stars, and the same $\left\langle M_{K}\right\rangle$-period relationship. Previous work which assumed the two groups of stars to behave identically is thus shown to be correct.
\end{abstract}

Subject headings: globular clusters: individual (M5, M92) - stars: variables: other (RR Lyr)

\section{INTRODUCTION}

The distances to globular clusters are usually determined by fixing the absolute magnitudes of their horizontal branch stars at the location of the RR Lyrae variable gap. The assumed value for the absolute luminosity of RR Lyrae variables rests upon statistical parallaxes for field RR Lyrae stars and on variations of the Baade-Wesselink (Baade 1926; Wesselink 1969) method for field RR Lyrae stars. Distances to globular clusters, which rely on main-sequence locations as defined by field subdwarfs such as in Sandage (1960) or Carney (1980) suffer from the serious limitation of the extremely small number for field subdwarfs which have accurate parallaxes, and of the need to include corrections to the luminosity for varying metallicities. Many of the field subdwarfs may be slightly evolved and thus have somewhat higher luminosities than the true main sequence.

These cluster distances are then used to determine cluster ages, and small errors in distance propagate into substantial age uncertainties. In addition, the absolute magnitudes of the RR Lyrae variables directly calibrate the extragalactic distance scale. RR Lyrae stars have been detected in the field and clusters of the Magellanic Clouds (see the review by Walker 1991), in M31 (Pritchet \& van den Bergh 1986), and in several other galaxies of the Local Group (Saha, Hoessel, \& Krist 1992 and references therein) and will undoubtedly be found in additional galaxies by the Hubble Space Telescope.

In recent years major efforts have been undertaken by several groups to improve the absolute magnitude determinations for field RR Lyrae variables. These include the reanalysis of the statistical parallax problem with improved astrometric data by two independent groups (Hawley et al. 1986; Barnes \& Hawley 1986; Strugnell, Reid, \& Murray 1986). Many people have explored the Baade-Wesselink method, and there is a growing consensus that the use of visual and infrared photometry at $K$ together provides the most accurate distance determinations for reasons reviewed in Jones et al. (1992). Basically this is because $K$ photometry, way out in the tail of the Rayleigh-Jeans distribution, when combined with visual photometry provides a very long and stable baseline for the determination of $T_{\text {eff }}$.

In addition the total flux emitted at $K$ is more independent of surface gravity, metallicity, and the effect of shocks than are fluxes at visual wavelengths. The extensive infrared BaadeWesselink analyses of the field RR Lyrae stars have been reviewed by Liu \& Janes (1991), who combine their own extensive data with that of Jones, Carney, \& Latham (1988), Cacciari et al. (1989), and Fernley et al. (1987, 1989). They derive a metallicity-absolute $V$-magnitude relationship and a periodabsolute $K$-magnitude relationship which must be regarded at this time as well established for field RR Lyrae stars.

Cohen \& Gordon (1987) began the application of the BaadeWesselink method to globular cluster stars, and that work is continued here. In the interim Liu \& Janes (1990) observed four stars in M4 using the infrared Baade-Wesselink method, while Storm et al. (1992) are working on two stars in M5 and two in M92. We continue our program by providing in this paper an infrared Baade-Wesselink analysis for two RR Lyrae variables in M92 and four in M5. In $\S 2$ we present the observational material. The technique of analysis is described in $\S 3$, while the results are given in $\S 4$. A summary of our conclusions is given in the final section.

\section{OBSERVATIONAL MATERIAL FOR THE GLOBULAR CLUSTER RR LYRAE VARIABLES}

The RR Lyrae variables discussed here are V1 and V6 in M92 and the same four variables in M5 as were analyzed by Cohen \& Gordon (1987) (V8, V12, V32, and V59). Infrared photometry of these stars is given in Cohen \& Matthews (1992), where visual photometry for these stars and six other globular cluster RR Lyrae stars is also presented. A discussion of the details of the period shifts is given there as well. Here we present the radial velocities for the M92 RR Lyrae variables; data for the M5 variables is given in Cohen \& Gordon.

The spectroscopic data are from the nights of 1987 April 15, 
16, and 17, 1987 May 6, 7, and 8, and 1988 April 25 and 26, using the double spectrograph at the Cassegrain focus of the $5 \mathrm{~m}$ Hale telescope at Palomar Mountain. Integrations were always 30 minutes long as a compromise between avoiding excessive blurring in phase and securing an adequate signal level for high-precision velocities from stellar spectra of lowmetallicity stars with few strong spectral features. Observations were carried out irrespective of seeing, transparency, or air mass (within the range up to $\sec [z]=1.9$ and absorption due to clouds of less than $1.0 \mathrm{mag}$ ). The detector was a $800 \times 800$ pixel TI CCD, and we used the $1200 \mathrm{~g} \mathrm{~mm}^{-1}$ grating in second order (with substantial vignetting occurring within the optical train) to yield a spectral resolution of $0.37 \AA$ pixel $^{-1}$. With a $1^{\prime \prime}$ slit the FWHM of arc lines is 2 pixels. Noble gas arcs were always taken before and after each exposure in the appropriate slit position. The spectral coverage extended from 5100 to $5400 \AA$, including the $\mathrm{Mg}$ triplet and various strong Fe-dominated blends such as $\lambda 5270$. Typical count rates were 1500 counts pixel $^{-1}$ (3000 electrons pixel $\left.{ }^{-1}\right)$; near maximum light for conditions near $1^{\prime \prime}$. Several bright template stars were observed each night.

Although the spectrograph entrance slit is over $2^{\prime}$ long, that part visible in the television guiding system is only $90^{\prime \prime}$ long. Since there are only nine RRab stars in M92 with periods from Sawyer Hogg (1973), three of which can be eliminated as they have the Blashko effect, it is fortunate that the pair M92-V1 and M92-V6 obey all the relevant criteria, and spectra of both of them could be obtained simultaneously by rotating the position angle of the slit on the sky.

The data were reduced using Figaro on the imageprocessing Vaxcluster of the Caltech astronomy department. After flattening, the single spectrum or pair of spectra (as well as a sky spectrum chosen from a clear region as free of stars as possible along the slit immediately adjacent to each object) was extracted to one dimension. Next we subtracted off the appropriate sky spectrum. The spectra were cross-correlated with the template spectrum for each night. The arcs were also crosscorrelated with the arc for the template star, arcs before each exposure and afterward being treated separately. This enabled the construction of a flexure table as a function of time during each night. The cross-correlation peaks, signal levels, and visual appearance of the $\mathrm{Mg}$ triplet of all the spectra were examined, and several radial velocities were rejected as unreliable. In general, these were from the beginning or end of the night, when the air mass approached 2.0 and the seeing deteriorated, so the signal level obtained in the maximum 30 minute integration was inadequate. (This was done prior to constructing the radial velocity-phase curves.) The zero point of our radial velocities assumes that the heliocentric radial velocity of $\mu$ Leo is $13.86 \mathrm{~km} \mathrm{~s}^{-1}$ and that of HD 122563 is $-24.7 \mathrm{~km} \mathrm{~s}^{-1}$. (These are the mean values for the modern determinations in Abt \& Biggs 1972.) The uncertainty is reviewed in Cohen \& Gordon (1987) and is derived as $4 \mathrm{~km}$ $\mathrm{s}^{-1}$.

The heliocentric radial velocities derived from the spectra are listed in Table 1 as a function of Julian day and phase (both given for the midpoint of the exposure) for the two RR Lyrae variables in M92. There are 43 independent radial velocity measurements for each star. The radial velocity-phase $(\phi)$ curves are plotted for the two stars in Figures 1 and 2. The uncertain measurements are indicated by crosses, while the most reliable measurements are shown as filled circles. Smooth curves were drawn through the $v_{r}-\phi$ curve for each star, and the mean velocities $v_{\gamma}$ were determined. These were -128.1 for
TABLE 1

M92 RR LyRae Radial Velocities

\begin{tabular}{|c|c|c|c|c|}
\hline \multirow[b]{2}{*}{$\begin{array}{l}\text { Julian Date } \\
(-2,440,000)\end{array}$} & \multicolumn{2}{|c|}{ M92-V1 } & \multicolumn{2}{|c|}{ M92-V6 } \\
\hline & Phase & $\left(\begin{array}{c}v_{r} \\
\left(\mathrm{~km} \mathrm{~s}^{-1}\right)\end{array}\right.$ & Phase & $\begin{array}{c}v_{r} \\
\left(\mathrm{~km} \mathrm{~s}^{-1}\right)\end{array}$ \\
\hline $6900.750 \ldots$ & 0.506 & -127.2 & 0.880 & \\
\hline $6901.750 \ldots$ & 0.892 & -106.3 & 0.503 & -119.6 \\
\hline $6901.750 \ldots$ & 0.928 & -128.1 & 0.546 & -111.7 \\
\hline $6901.750 \ldots$ & 0.964 & -138.6 & 0.587 & -125.0 \\
\hline $6902.000 \ldots$ & 0.057 & -154.7 & 0.696 & -121.5 \\
\hline $6902.000 \ldots$ & 0.090 & -154.0 & 0.735 & -116.7 \\
\hline $6902.000 \ldots$ & 0.125 & -154.7 & 0.776 & -113.6 \\
\hline $6902.000 \ldots$ & 0.159 & -153.6 & 0.817 & -120.0 \\
\hline $6902.750 \ldots$ & 0.308 & -138.4 & 0.163 & -160.1 \\
\hline $6902.750 \ldots$ & 0.343 & -130.0 & 0.203 & -152.5 \\
\hline $6902.750 \ldots$ & 0.379 & -130.6 & 0.245 & -142.9 \\
\hline $6903.000 \ldots$ & 0.413 & -124.8 & 0.285 & -138.2 \\
\hline $6903.000 \ldots$ & 0.463 & -133.2 & 0.343 & -139.9 \\
\hline $6903.000 \ldots$ & 0.507 & -121.6 & 0.395 & -126.9 \\
\hline $6903.000 \ldots$ & 0.544 & -113.2 & 0.438 & -125.3 \\
\hline $6903.000 \ldots$ & 0.580 & -112.4 & 0.481 & -130.2 \\
\hline $6921.750 \ldots$ & 0.270 & -138.0 & 0.744 & \\
\hline $6921.750 \ldots$ & 0.308 & -137.9 & 0.788 & -121.0 \\
\hline $6921.750 \ldots$ & 0.346 & -134.4 & 0.833 & -117.2 \\
\hline $6921.750 \ldots$ & 0.394 & -133.5 & 0.888 & -108.2 \\
\hline $6921.750 \ldots \ldots \ldots$ & 0.429 & -132.0 & 0.930 & -132.9 \\
\hline $6922.000 \ldots \ldots \ldots$ & 0.466 & -127.7 & 0.973 & -143.7 \\
\hline $6922.000 \ldots$ & 0.503 & -127.5 & 0.016 & -157.8 \\
\hline $6922.000 \ldots$. & 0.582 & -116.9 & 0.108 & -165.4 \\
\hline $6922.750 \ldots$ & 0.754 & -108.9 & 0.482 & -128.3 \\
\hline $6922.750 \ldots \ldots \ldots$ & 0.795 & -111.5 & 0.530 & -128.9 \\
\hline $6922.750 \ldots$ & 0.842 & -109.8 & 0.585 & -114.6 \\
\hline $6923.000 \ldots$. & 0.878 & -106.7 & 0.627 & -125.1 \\
\hline $6923.000 \ldots$ & 0.914 & -119.6 & 0.668 & -121.4 \\
\hline $6923.000 \ldots \ldots \ldots$ & 0.955 & -156.8 & 0.717 & -129.4 \\
\hline $6923.750 \ldots \ldots \ldots$ & 0.130 & & 0.093 & -164.6 \\
\hline $7276.750 \ldots$ & 0.517 & -119.3 & 0.550 & -110.3 \\
\hline $7276.750 \ldots \ldots \ldots$ & 0.560 & -113.1 & 0.600 & -108.8 \\
\hline $7277.000 \ldots \ldots \ldots$ & 0.603 & -108.9 & 0.651 & -105.3 \\
\hline $7277.000 \ldots \ldots \ldots$ & 0.644 & -107.0 & 0.699 & \\
\hline $7277.000 \ldots \ldots \ldots$ & 0.681 & -105.7 & 0.743 & -109.1 \\
\hline $7277.000 \ldots \ldots \ldots$ & 0.720 & -106.6 & 0.788 & -107.4 \\
\hline $7277.750 \ldots \ldots \ldots$ & 0.940 & -139.8 & 0.217 & -138.0 \\
\hline $7277.750 \ldots \ldots \ldots$ & 0.981 & -142.4 & 0.265 & -139.1 \\
\hline $7278.000 \ldots \ldots \ldots$ & 0.020 & -145.9 & 0.311 & -129.8 \\
\hline $7278.000 \ldots \ldots \ldots$ & 0.060 & -146.2 & 0.357 & -122.7 \\
\hline $7278.000 \ldots \ldots \ldots$ & 0.098 & -144.7 & 0.402 & -121.3 \\
\hline $7278.000 \ldots \ldots \ldots$ & 0.136 & -141.2 & 0.446 & -113.5 \\
\hline
\end{tabular}

M92-V1 and $-132.7 \mathrm{~km} \mathrm{~s}^{-1}$ for M92-6. The $v_{\gamma}$ determined by Storm et al. (1992) for M92-V1 is $-125.4 \mathrm{~km} \mathrm{~s}^{-1}$, in good agreement with our determination, while the systemic radial velocity for M92 compiled by Webbink (1981) is - 120.5 with a dispersion of $6.7 \mathrm{~km} \mathrm{~s}^{-1}$. The M5 $v_{\gamma}$ velocities of Cohen \& Gordon (1987) lie very close to the systemic velocity of M5 given by Webbink.

\section{DERIVATION OF DISTANCES AND MEAN RADII}

\subsection{Method of Analysis}

The analysis of this data has as its goal the derivation of the angular diameter (henceforth $\theta$ ) as a function of phase from both the radial velocity and photometric data sets. Forcing agreement between these two $\theta(\phi)$ relationships determines the distance to the pulsating variable star. The manipulation of the radial velocities is straightforward. Liu (1991) has shown that the radial velocity curves of field RR Lyrae stars are simply a single curve scaled by the velocity amplitude appropriate to each variable. We fit a curve of this form to each of the globu- 


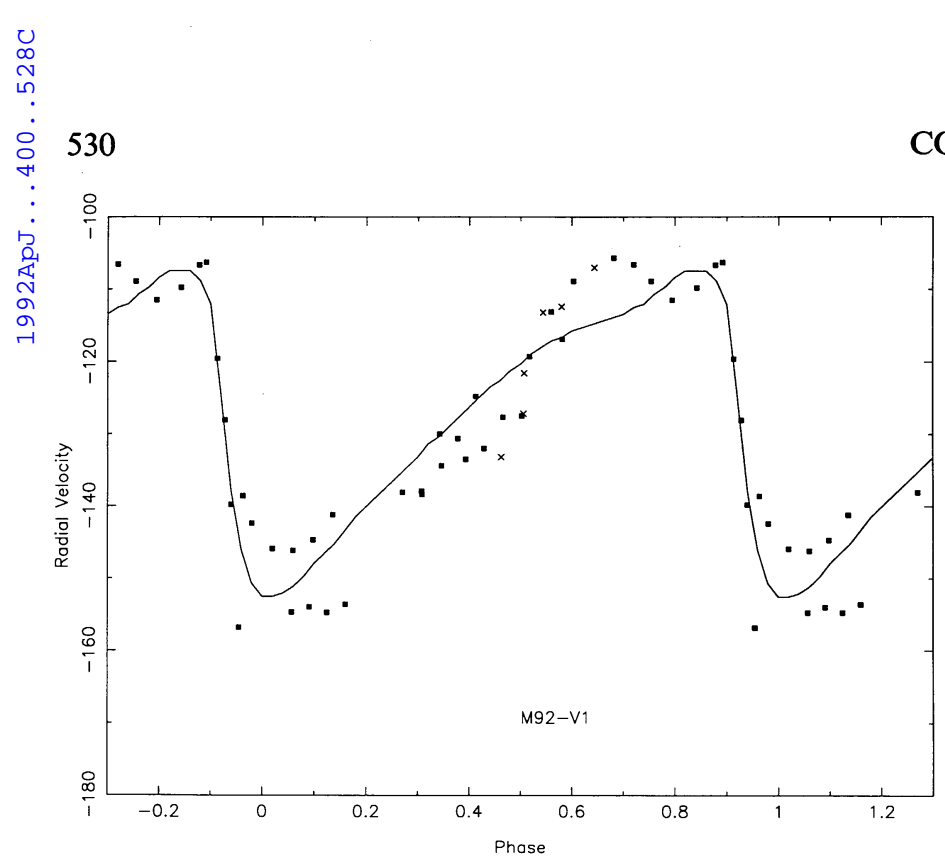

FIG. 1.-Heliocentric radial velocity for RR Lyrae M92-V1 as a function of phase. Crosses: the least accurate points; filled circles: the most reliable measurements; solid curve: fit to the data using Liu's (1990) synthetic velocity curve.

lar cluster RR Lyrae variables. The solid lines in Figures 1 and 2 represent such fits, which have been digitized at 0.02 cycle intervals. Note that only the relative radial velocities matter here, which makes the use of single velocity curve scaled by the velocity amplitude to represent the globular cluster RR Lyrae variables feasible.

The pulsational velocity is the difference between $v_{r}$ and $v_{\gamma}$, defined in $\S 2.1$, multiplied by the projection factor $p$ to convert a line-of-sight velocity into a pulsational velocity. This factor has been set at 1.31 . Values adopted in recent analyses range from 1.30 to 1.35 ; the choice of $p$ and the accuracy with which it is known are discussed in Hindsley \& Bell (1986). If $p$ is underestimated by $5 \%$, the distance to the pulsating variable star will be underestimated by $5 \%$.

The pulsational velocity is then integrated over time (i.e., phase times the period of the variable) to yield the difference in radius $\delta R(\phi)$. We begin the integration just past maximum

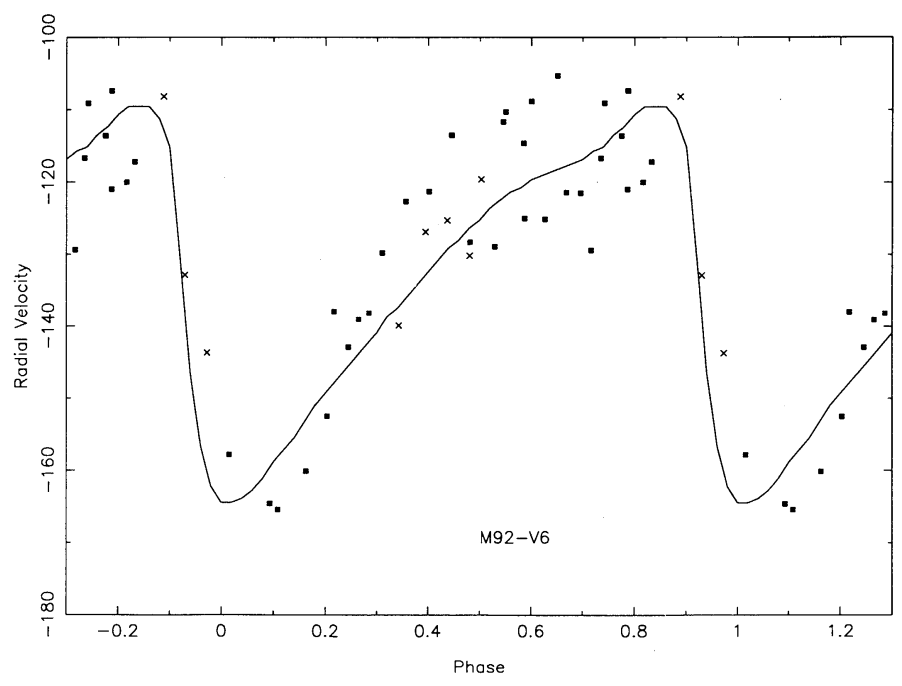

FIG. 2.-Same as Fig. 1 for RR Lyrae variable M92-V6

light when the star begins to expand, as that puts the worst determined part of the pulsational velocity curve, that of the rapid drop in pulsational velocity at maximum light, at the end of the integration. If a value for the radius at $\phi=0$ and one for the distance to the globular cluster are assumed, the angular diameter over the cycle is easily obtained.

As pointed out by Oke, Giver, \& Searle (1962), ideally one wants to measure a radial velocity from a feature arising at the same optical depth where the continuum is formed; this is clearly impossible. We have used the $\mathrm{Mg}$ triplet region, which in these metal-poor stars contains only relatively weak absorption lines. Jones et al. (1987) and Saha \& White (1990) have demonstrated that there is no detectable gradient of velocity with depth over the relevant range of optical depth. We are thus confident that the observed radial velocity curve is a true reflection of the pulsational velocity as a function of phase.

The derivation of the $\theta-\phi$ relationship from the photometric data has evolved from the scheme of Cohen \& Gordon (1987). Basically the Kurucz (1979) model atmospheres are used to define the expected stellar fluxes as a function of $T_{\text {eff }}$, surface gravity, and metallicity. The absolute calibration of the observed magnitude systems are given by Oke \& Schild (1970) and by Oke $\&$ Gunn (1983) for $i$, assuming an effective wavelength of $8100 \AA$ for the $i$ filter. The absolute calibration for $J$ and $K$ is that used by the Caltech infrared group as given in Neugebauer et al. (1987). It carries a 5\% uncertainty, much larger than that of the absolute flux calibration at optical wavelengths. The conversion between observed colors and colors predicted from the Kurucz model atmosphere grid is given by the model atmospheres analysis by Bell \& Oke (1986) of the bright metal-poor subdwarfs that define the ThuanGunn system and also serve as spectrophotometric standards, with infrared photometry from Carney $(1983 a, b)$ to define the zero point of the $V-K$ and $V-J$ colors. The surface gravity$\phi$ relationship for RR Lyrae variables is taken from Manduca et al. (1981) assuming a mass of $0.6 M_{\odot}$. The fitting procedure used to determine the best choice of distance and radii is that described by Cohen \& Gordon.

Note that there are many possibilities here. $T_{\text {eff }}$ can be defined from the $V-i, V-J$, or $V-K$ colors, and the absolute flux can be defined at any of the four colors. Unless otherwise specified, for the reasons advanced in $\S 1$, we use $(V-K)(\phi)$ to determine $T_{\text {eff }}(\phi)$ and $K(\phi)$ to determine the absolute flux. This choice gives the smoothest photometric $\theta(\phi)$ curve, with the best agreement between $\theta\left(v_{r}\right)-\phi$ and $\theta$ (photometric) $-\phi$, and no sign of phase shifts.

This method of analysis was tested by successfully reproducing Liu's (1990) Baade-Wesselink analysis of several field RR Lyrae variables. The distances obtained were identical to his in all cases to within $3 \%$.

\section{RESULTS}

\subsection{Distances and Radii}

We apply the procedure described in $\S 3$ to carry out a Baade-Wesselink analysis for the globular cluster RR Lyrae variables in M5 and M92. The infrared and optical photometry was given in Cohen \& Matthews (1992), and the radial velocities are given in $\S 2$. The digitization of the mean light curves in intervals of 0.02 cycles is straightforward, while the fitting of the velocity curve by a synthetic function was described earlier. As in Cohen \& Gordon (1987), a restricted phase interval of $0.2-0.8$ was used to avoid the incidence of shocks around the epoch of minimum radius. 


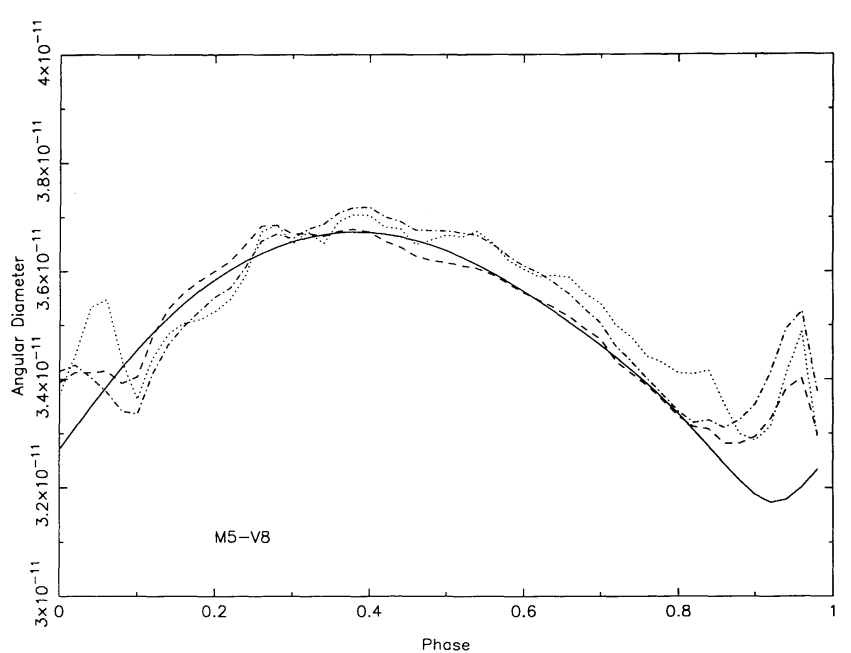

Fig. 3.-Angular diameter $(\theta)$ is shown as a function of phase $(\phi)$ for the RR Lyrae M5-V8. Solid curve: $\theta(\phi)$ deduced from integrating the radial velocity curve; other curves: deduced from the photometric $\theta(\phi)$ determinations using the $K$-magnitude to determine the absolute flux. Dashed line: $T_{\text {eff }}$ deduced from $V-K$ colors; dot-dashed line: uses $V-J$ colors, while the dotted line uses $V-i$ colors.

The metallicities of the two globular clusters are taken from Zinn (1985), while the reddening adopted for M92 of $0.02 \mathrm{mag}$ is from Stetson \& Harris (1988) and that of M5 (0.03 mag) from Zinn (1980).

The fitting procedure converged rapidly and was very straightforward. The best fit for each star is shown in Figures 3-8. In all cases the solid curve is the integration of the radial velocity curve, while the remaining lines depict the photometric data. In each case, the $K$-magnitudes determine the absolute flux, while $T_{\text {eff }}$ is determined from the $V-K$ colors for the dashed line, from $V-i$ for the dotted line, and from $V-J$ for the dot-dashed line. The final fits all use $V-K$ colors to determine $T_{\text {eff }}$. In examining these figures, bearing mind the restricted phase interval, one is struck by the relatively good fits that have been achieved using the $V-K$ color and by the absence of the apparent phase shift manifested in BaadeWesselink analyses that rely on blue colors, such as those of Cacciari et al. (1989) or Cohen \& Gordon (1987).

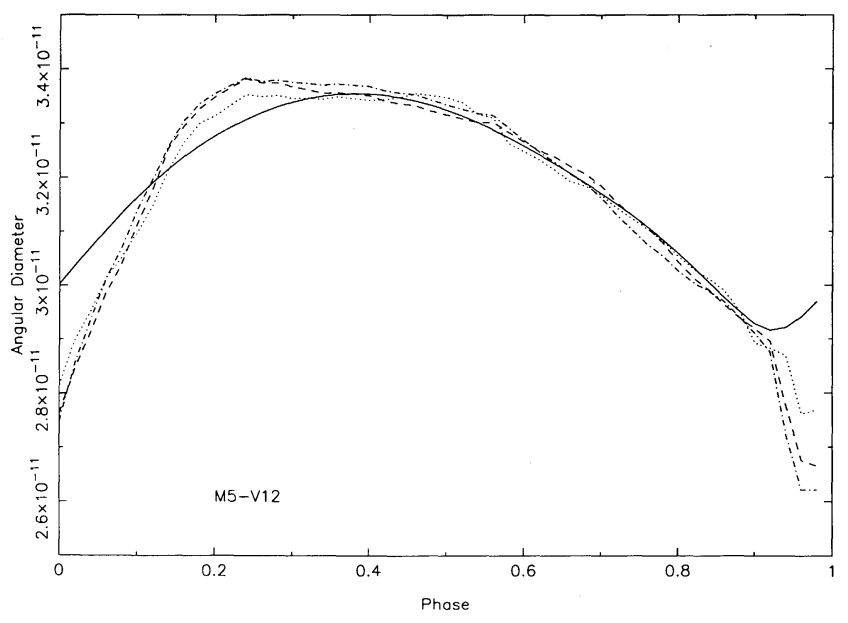

FIG. 4.-Same as Fig. 3 for RR Lyrae M5 - V12

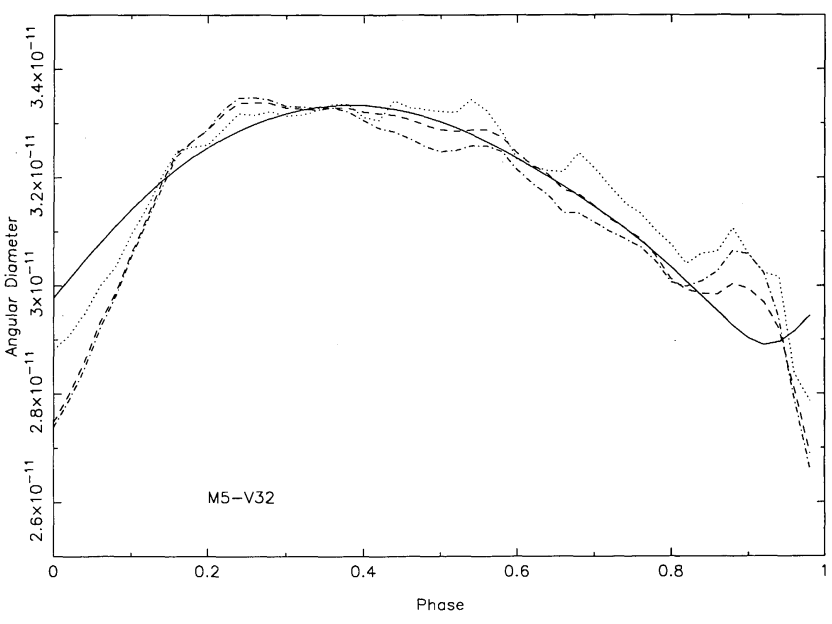

Fig. 5.-Same as Fig. 3 for RR Lyrae M5 - V32

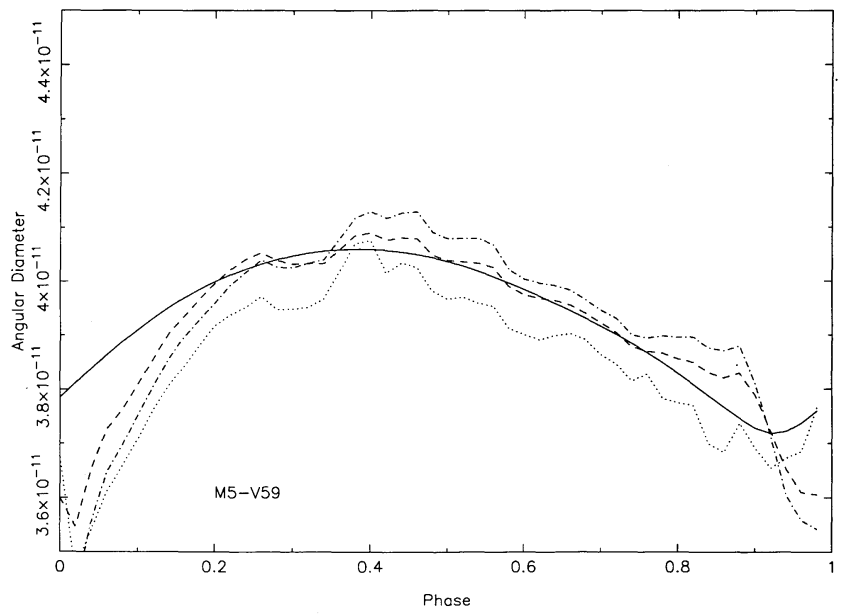

FIG. 6. - Same as Fig. 3 for RR Lyrae M5 - V59

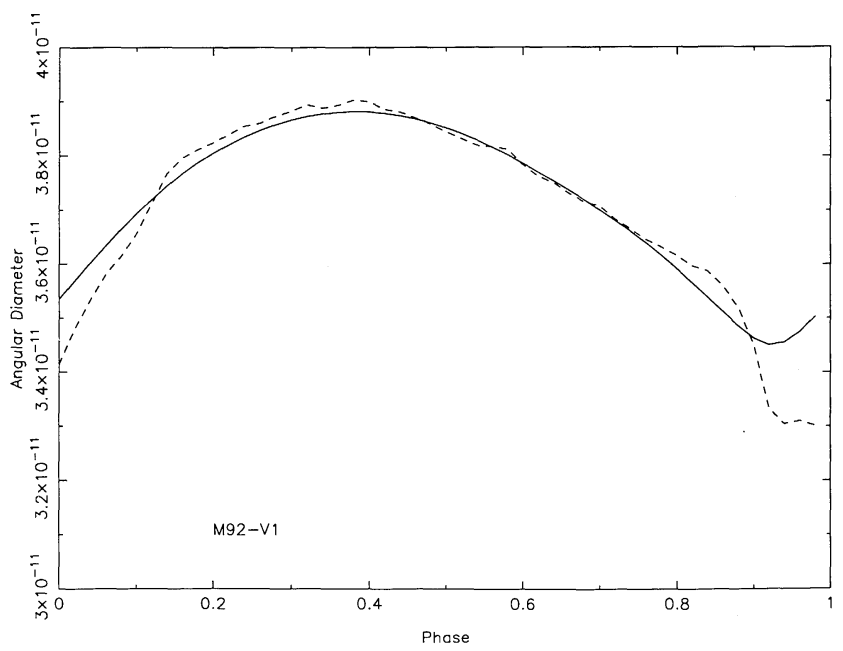

FIG. 7.-Same as Fig. 3 for RR Lyrae M92-V1 


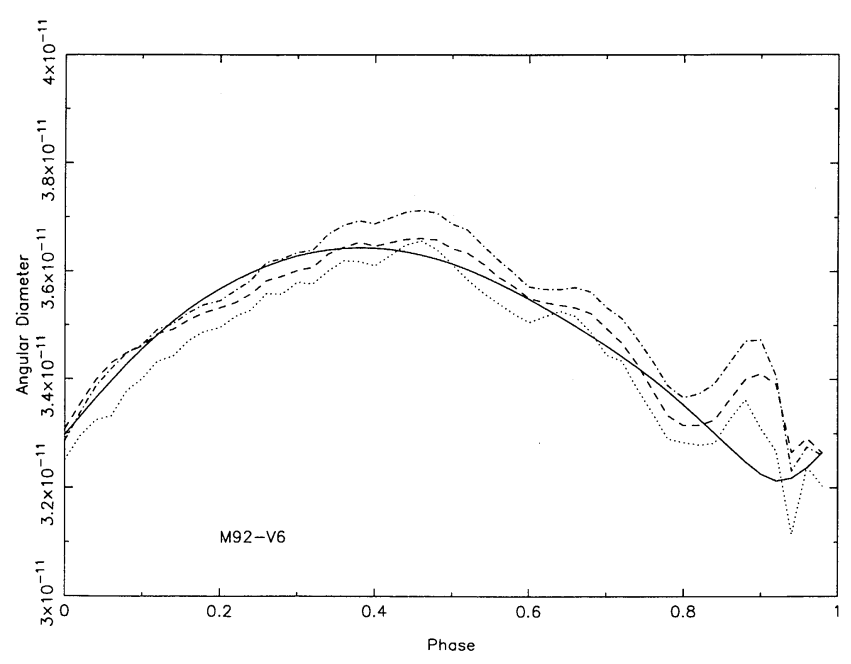

FIG. 8.-Same as Fig. 3 for RR Lyrae M92 - V6

Table 2 contains the best-fitting distance, the mean radius of the star, and the range over which the radius varies, as well as the radial velocity amplitude, used in specifying the synthetic radial velocity curve. The previous distance for M5 from Richer \& Fahlman (1987) was based on fitting the local subdwarfs to the lower main sequence and gave $(m-M)_{\mathrm{app}}=$ $14.30 \pm 0.20$, in good agreement with our result of $14.194+0.093\left(=A_{V}\right)=14.287$. Stetson \& Harris (1988) follow a slightly different tack, using the theoretical isochrones as intermediaries to refer the cluster sequence to the field subdwarfs. They derive $(m-M)_{\mathrm{app}} \approx 14.60$ for M92. We derive $(m-M)_{\mathrm{app}}=14.474+0.062\left(=A_{V}\right)=14.536$. Thus agreement with previous high-quality measurements is very good, well within the uncertainty of the measurements.

The uncertainty of each distance has several components. The first is that contributed by $v_{r}$ errors, and, being very conservative, we have described them by the change in fit when the amplitude of the velocity curve is altered by $3 \mathrm{~km} \mathrm{~s}^{-1}$, namely $\pm 0.5 \mathrm{kpc}$. Then come the uncertainties induced by errors in the $V$ - and $K$-photometry. The effect of altering the amplitude of the variation over the cycle at $K$ by $0.03 \mathrm{mag}$ is to produce a distance change of $1.0 \mathrm{kpc}$. Note that the effect of a zero-point error in the $K$-magnitude scale of the same size is much smaller. The infrared photometry is less extensive for the M5 stars than for the M92 stars, so we take an error of $\pm 0.67 \mathrm{kpc}$ for the M5 stars and $\pm 0.33 \mathrm{kpc}$ for the M92 stars. The effect of errors in the $V$-photometry is taken as $\pm 0.3 \mathrm{kpc}$ for all the stars. Finally, there is a systematic error arising from the uncer-

TABLE 2

Distances and Radil for Globular Cluster RR Lyrae Variables

\begin{tabular}{ccccc}
\hline \hline Star & $\begin{array}{c}\text { Velocity } \\
\text { Amplitude } \\
\left(\mathrm{km} \mathrm{s}^{-1}\right)\end{array}$ & $\begin{array}{c}\text { Mean Radius } \\
\left(10^{6} \mathrm{~km}\right)\end{array}$ & $\begin{array}{c}\delta(R) \\
\left(10^{6} \mathrm{~km}\right)\end{array}$ & $\begin{array}{c}\text { Distance } \\
(\mathrm{kpc})\end{array}$ \\
\hline M5 & & & & \\
V8 $\ldots \ldots \ldots \ldots$ & 57 & 3.48 & 0.492 & 6.4 \\
V12 $\ldots \ldots \ldots \ldots$ & 62 & 3.38 & 0.459 & 6.8 \\
V32 $\ldots \ldots \ldots \ldots$. & 65 & 3.40 & 0.471 & 6.9 \\
V59 $\ldots \ldots \ldots \ldots$ & 46 & 4.58 & 0.394 & 7.5 \\
M92 & & & & \\
V1 $\ldots \ldots \ldots \ldots$. & 46 & 4.46 & 0.512 & 7.7 \\
V6 $\ldots \ldots \ldots \ldots$. & 56 & 4.31 & 0.476 & 8.0 \\
\hline
\end{tabular}

tainty in the absolute flux calibration at $K$ of $\pm 5 \%$ given by Neugebauer et al. (1987). The uncertainty in the $V$ absolute flux calibration is small by comparison. There is also the systematic error in the choice of the velocity projection factor $p$ to which we ascribe a value of $3 \%$. Thus our final distances are $7.85 \pm 0.45 \mathrm{kpc}$ for M92, and $6.9 \pm 0.45 \mathrm{kpc}$ for M5, with an additional systematic uncertainty of $0.3 \mathrm{kpc}$.

\subsection{Comparison with the Field RR Lyrae Relationships}

Collecting the extensive data for field RR Lyrae variables described in $\S 1$, Jones et al. (1992) have summarized the two relationships that appear. The first is the absolute visual magnitude-metallicity relationship,

$$
\left\langle M_{V}\right\rangle=0.16( \pm 0.03)[\mathrm{Fe} / \mathrm{H}]+1.02( \pm 0.03) \text {. }
$$

The second is an infrared period-luminosity law of the form

$$
\left\langle M_{K}\right\rangle=-2.33( \pm 0.20) \log \left(P_{0}\right)-0.88( \pm 0.06) .
$$

Relationships similar to these, with slightly different numerical coefficients, can be derived from pulsation theory (see Lee 1990 or van Albada \& Baker 1971). We now ask, how well do these globular cluster variables obey these relationships? Figures 9 and 10 show the field stars together with the M5 and M92 RR Lyrae stars. The open circles denote the field RR Lyrae variables, while the filled circles indicate the globular cluster variables, including data from Liu \& Janes (1990) on M4. The crosses in Figure 10 indicate the c-type RR Lyrae field stars; the $P_{0} / P_{1}$ relation of van Albada \& Baker (1971) was used. Data from Cohen \& Matthews (1992) on additional RR Lyrae stars in M92 and M5 were added to Figure 9.

The agreement with equation (1) is very good; the M92 points lie 0.09 mag brighter than expected, while in the mean in M5 points lie 0.02 mag fainter than expected. Considering the errors on the distances, this agreement is more than satisfactory. In comparison with equation (2), the M92 stars lie 0.18 too bright, while the M5 stars in the mean are $0.11 \mathrm{mag}$ too bright. One has the impression that the scatter about equation (2) is significantly larger than that about equation (1).

One possibility is that this scatter may be due to the effect of

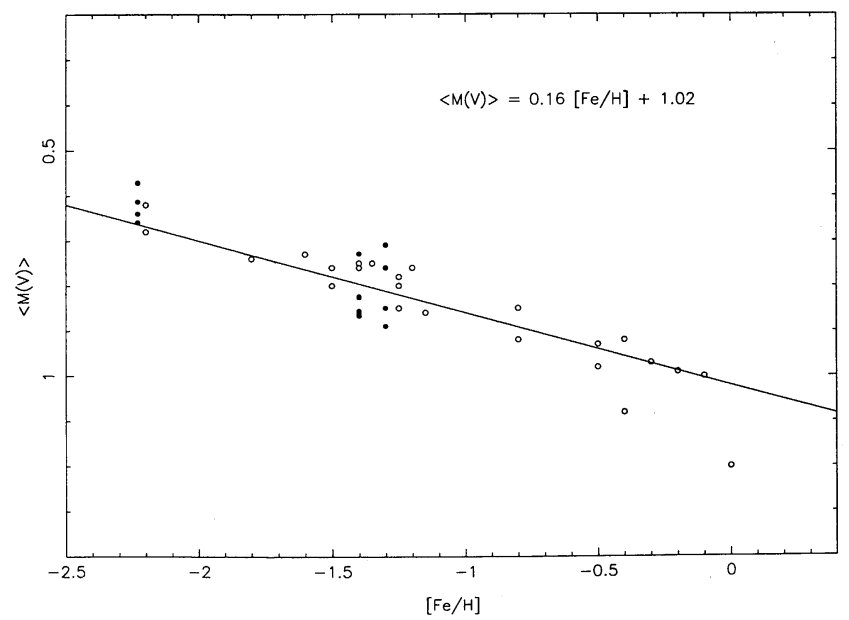

Fig. 9.-Relationship between $\left\langle M_{V}\right\rangle$ and $[\mathrm{Fe} / \mathrm{H}]$ is shown for field RR Lyrae stars (open circles) and globular cluster RR Lyrae stars (filled circles). The field star data is that compiled by Liu \& Janes (1991). The globular cluster data include the analysis of four stars in M4 by Liu \& Janes (1990). The best-fit line is from Jones et al. (1992). 


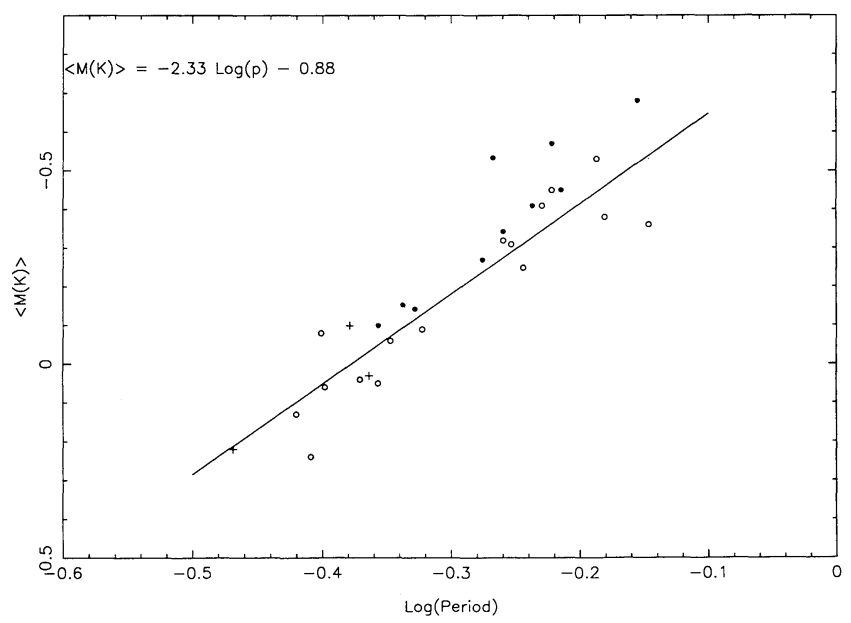

FIG. 10.-The relationship between $\left\langle M_{K}\right\rangle$ and (fundamental) period is shown for field RR Lyrae stars (open circles) and globular cluster stars (filled circles). The crosses denote field c-type RR Lyrae stars. The sources of data are the same as in Fig. 9.

stars evolving off the zero-age horizontal branch. The most discrepant star is M5-V59, which is bright in $V$ (by $0.1 \mathrm{mag}$ ) and much brighter at $K$ (by $0.27 \mathrm{mag}$ ) than expected. Sandage (1990) has studied the vertical height of the horizontal branch at $V$ and found that it reaches $0.2 \mathrm{mag}$ in the metal-poor clusters and climbs to $0.6 \mathrm{mag}$ in a globular cluster like M4. At $K$, as shown by the data of Longmore et al. (1990), the vertical height of the horizontal branch is huge, reaching 0.6 mag even in the metal-poor clusters, but most of this is taken up by the period dependence of equation (2). Combining the data of Sandage and of Longmore et al. for the clusters in common with many variables (M3 and NGC 3201), there is suggestive, but not conclusive, evidence that RR Lyrae stars which have evolved off the ZAHB are too bright compared to the expectations of both equations (1) and (2). Note that two apparently highly evolved field RR Lyrae stars, DX Del and SS Leo, were omitted from consideration by Jones et al. (1992), but these two stars show large deviations at $V$ as well as at $K$ between the observed magnitudes and those predicted by equations (1) and (2), whereas we see deviations mainly at $K$. They may simply be more extreme cases of the same phenomenon.

\subsection{Implications for Globular Cluster Ages}

One of our major conclusions is the globular cluster RR Lyrae stars behave just like field RR Lyrae variables. To within the uncertainties, they obey identical absolute $V$-magnitudemetallicity and absolute $K$-magnitude-period relationships. This is not entirely unexpected, since the use of a mean velocity curve, derived from field stars, must contribute to that result at some level.

Thus all the previous work in the field remains valid. In particular, Carney, Storm, \& Jones (1992) discuss again the globular cluster ages using the difference in magnitude between the apparent magnitude of the horizontal branch and the main-sequence turnoff from Buonanno, Corsi, \& Fusi Pecci (1989) as the age indicator and the apparent magnitude of the horizontal branch combined with equation (1) as the distance indicator. Their conclusions depend on the adopted value of $[\mathrm{O} / \mathrm{Fe}]$ as a function of $[\mathrm{Fe} / \mathrm{H}]$, but if the results of Abia $\&$ Rebolo (1989) are adapted, then the mean age of globular clusters at a given metallicity is a constant, approximately $14 \times 10^{9} \mathrm{yr}$, independent of metallicity.

\section{SUMMARY}

We have presented an infrared Baade-Wesselink analysis for two stars in the extremely metal-poor globular cluster M92 and four in the intermediate metallicity cluster M5. The visual and infrared photometry were presented in Cohen \& Matthews (1992), while in $\S 2$ we present the radial velocity measurements. The method of analysis used relies on the theoretical model atmospheres of Kurucz (1979) and the absolute flux calibration of the observed $V, i, J$, and $K$-magnitudes. The best results are obtained using the $V-K$ colors to derive $T_{\text {eff }}$ and the $K$-magnitudes to deduce the absolute flux. Under such circumstances the smoothest possible $\theta$-phase relationships, where $\theta$ is the angular diameter, are obtained, with no sign of phase shifts. Thus the maximum range in phase can be used to match the $\theta$-phase relationship derived from integrating the radial velocity-phase curve to that from the photometric data. We obtain the distance to M5 as $6.9 \pm 0.45 \mathrm{kpc}$, and that to M92 as $7.85 \pm 0.45 \mathrm{kpc}$, with a $0.3 \mathrm{kpc}$ additional systematic uncertainty. These results are in excellent agreement with the previously published distances, which are based on fitting the globular cluster main sequence to the subdwarf field stars of known distances. With these distances we obtain an absolute mean magnitude corrected for reddening $M_{V}^{0}$, of $0.86 \pm 0.14$ mag for the M5 RR Lyrae variables, while $M_{V}^{0}=0.62 \pm 0.11$ for the M92 RR Lyrae variables.

The globular cluster RR Lyrae variables behave just like the field RR Lyrae stars. They obey the same $\left\langle M_{V}\right\rangle-[\mathrm{Fe} / \mathrm{H}]$ relationship and the same $\left\langle M_{K}\right\rangle$-period relationship as do the field RR Lyrae stars. There is the same occasional exception of a star which is brighter than expected, which probably is the manifestation of stars which have evolved off the zero age horizontal branch.

In the opinion of this author, at this time one should consider not pressing onward with further Baade-Wesselink analyses of globular cluster stars. They are too faint, and the observational effort is great, while the final errors in distance will never be much less than $10 \%$ per star. This effort would be better spent on improving the field star sample with accurate distances determined by the infrared Baade-Wesselink method.

I am grateful to Karen Lassey (a SURF student at Caltech) for help during the initial stages of this work. I am grateful to Gaston Araya Machining and the Caltech Recycling Center for financial support.

\section{REFERENCES}

Abia, C., \& Rebolo, R. 1989, ApJ, 347, 186

Abt, H. A., \& Biggs, E. S. 1972, Bibliography of Stellar Radial Velocities (New York: Latham Process)

Baade, W. 1926, Astron. Nach., 228, 359

Barnes, T. G.., III, \& Hawley, S. L. 1986, ApJ, 307, L9

Bell, R. A., \& Oke, J. B. 1986, ApJ, 307, 253

Buonanno, R., Corsi, C. E., \& Fusi Pecci, F. 1989, A\&A, 216, 80

Cacciari, C., Clementini, G., Prevot, L., \& Buser, R. 1989, A\&A, 209, 141

Carney, B. W. 1980, ApJS, 42, 481
Carney, B. W. 1983a, AJ, 88, 610

Carney, B. W., Storm, J., \& Jones, R. V. 1992, ApJ, 386, 663

Cohen, J. G., \& Gordon, G. A. 1987, ApJ, 318, 215

Cohen, J. G., \& Matthews, K. 1992, PASP, submitted

Fernley, J. A., Longmore, A. J., Jameson, R. F., Watson, F. G., \& Wesselink, T. 1987, MNRAS, 226, 927

Fernley, J. A., Lynas-Gray, A. E., Skillen, I., Jameson, R. F., Marang, F., Kilkenny, D., \& Longmore, A. J. 1989, MNRAS, 236, 447 


\section{COHEN}

Hawley, S. L., Jeffreys, W. H., Barnes, T. G., III, \& Wan Lei, 1986, ApJ, 302, 626

Hindsley, R., \& Bell, R. A. 1986, PASP, 98, 881

IN. Jones, R. V., Carney, B. W., \& Latham, D. W. 1988, ApJ, 332, 206

IGI Jones, R. V., Carney, B. W., Latham, D. W., \& Kurucz, R. L. 1987, 312, 254

III Jones, R. V., Carney, B. W., Storm, J., \& Latham, D. W. 1992, ApJ, 386, 646

Kurucz, R. L. 1979, ApJS, 40,

Lee, Y. W. 1990, ApJ, 363, 159

Liu, T. 1990, Ph.D. thesis, Boston Univ. 1991, PASP, 103, 205

Liu, T. X., \& Janes, K. A. 1990, ApJ, 360, 561

1991, in The Formation and Evolution of Star Clusters, ed. K. Janes (ASP Conf. Ser., 13)

Longmore, A. J., Dixon, R., Skillen, I., Jameson, R. F., \& Fernley, J. A. 1990, MNRAS, 247, 684

Manduca, A., Bell, R. A., Barnes, T. G., III, Moffett, T. J., \& Evans, D. S. 1981, ApJ, 250, 312

Neugebauer, G., Green, R. F., Matthews, K., Schmidt, M., Soifer, B. T., \& Bennett, J. 1987, ApJS, 63, 615

Oke, J. B., Giver, L. P., \& Searle, L. 1962, ApJ, 136, 393

Oke, J. B., \& Gunn, J. E. 1983, ApJ, 266, 713
Oke, J. B., \& Schild, R. E. 1970, ApJ, 161, 1015

Pritchet, C. S., \& van den Bergh, S. 1987, ApJ, 316, 517

Richer, H. B., \& Fahlman, G. 1987, ApJ, 316, 189

Saha, A., Hoessel, J. G., \& Krist, J. 1992, AJ, 103, 84

Saha, A., \& White, R. E. 1990, PASP, 102, 148

Sandage, A. R. 1970, ApJ, 162, 841

. 1990, ApJ, 350, 603

Sawyer Hogg, H. 1973, Publ. David Dunlop Obs., 3,

Stetson, P. B., \& Harris, W. E. 1988, AJ, 96, 909

Storm, J., Carney, B. W., Latham, D. W., Davis, R. J., \& Laird, J. B. 1992 PASP, 105, 168

Strugnall, P., Reid, N., \& Murray, C. 1986, MNRAS, 220, 413

Thuan, T. X., \& Gunn, J. E. 1976, PASP, 88, 543

Walker, A. R. 1991, in The Magellanic Clouds, ed. R. Haynes \& D. Milne (Boston: Kluwer), 307

Webbink, R. F. 1981, ApJS, 45, 229

Wesselink, A. J. 1969, MNRAS, 144, 297

van Albada, T. S., \& Baker, N. 1971, ApJ, 169, 31

Zinn, R. J. 1980, ApJS, 42, 19 1985, ApJ, 293, 424 